\title{
Prostaglandin-mediated inhibition of serotonin signaling controls the affective component of inflammatory pain
}

\author{
Anand Kumar Singh, Joanna Zajdel, Elahe Mirrasekhian, Nader Almoosawi, Isabell Frisch, Anna M. Klawonn, Maarit Jaarola, \\ Michael Fritz, and David Engblom
}

Department of Clinical and Experimental Medicine, Linköping University, Linköping, Sweden.

\begin{abstract}
Pain is fundamentally unpleasant and induces a negative affective state. The affective component of pain is mediated by circuits that are distinct from those mediating the sensory-discriminative component. Here, we have investigated the role of prostaglandins in the affective dimension of pain using a rodent pain assay based on conditioned place aversion to formalin injection, an inflammatory noxious stimulus. We found that place aversion induced by inflammatory pain depends on prostaglandin $E_{2}$ that is synthesized by cyclooxygenase 2 in neural cells. Further, mice lacking the prostaglandin $E_{2}$ receptor $E_{3}$ selectively on serotonergic cells or selectively in the area of the dorsal raphe nucleus failed to form an aversion to formalininduced pain, as did mice lacking the serotonin transporter. Chemogenetic manipulations revealed that $\mathrm{EP}_{3}$ receptor activation elicited conditioned place aversion to pain via inhibition of serotonergic neurons. In contrast to their role in inflammatory pain aversion, $\mathrm{EP}_{3}$ receptors on serotonergic cells were dispensable for acute nociceptive behaviors and for aversion induced by thermal pain or а к opioid receptor agonist. Collectively, our findings show that prostaglandin-mediated modulation of serotonergic transmission controls the affective component of inflammatory pain.
\end{abstract}

\section{Introduction}

Pain is an adaptive warning signal under physiological conditions, but it also causes a lot of suffering during various pathological states. Pain is a complex phenomenon with many components. The sensory component provides information about location, intensity, and quality of the painful event (1). In addition, pain is inherently unpleasant and induces a negative affective state $(2,3)$. Converging evidence indicates that the brain regions mediating the sensory and the affective components of pain are, at least partly, distinct $(1,4-6)$.

Prostaglandins are key regulators of nociceptive processing during inflammation, and prostaglandin synthesis inhibitors in the form of nonsteroidal antiinflammatory drugs are widely used for their analgesic properties. Prostaglandins modulate nociceptive signaling at many levels of the neuraxis (7), but it is unclear how they are involved in the affective dimension of pain.

\section{Results and Discussion}

To investigate the affective component of pain in mice, we used conditioned place avoidance induced by inflammatory pain. This test, in which mice learn to avoid a chamber where they experienced pain induced by formalin injection to the hind paw, has been used extensively to investigate the affective component of pain in rodents $(3,5,6,8)$. To explore the role of prostaglandins, we first interfered with the cyclooxygenases COX1 and COX2 (also known as PTGS1 and PTGS2), enzymes responsible for

Conflict of interest: The authors have declared that no conflict of interest exists. Submitted: September 15, 2016; Accepted: January 17, 2017. Reference information: / Clin Invest. 2017;127(4):1370-1374. https://doi.org/10.1172/JCI90678. prostaglandin synthesis. Normal (WT) mice, mice lacking COX1, and mice pretreated with a COX1 selective inhibitor (sc560, 5 $\mathrm{mg} / \mathrm{kg}$, i.p.; Figure 1B) avoided the chamber paired with formalin injections (Figure 1, A and B; for figures showing the behavioral experiments with individual values indicated, see Supplemental Figure 1; supplemental material available online with this article; https://doi.org/10.1172/JCI90678DS1). In contrast, the aversion was completely blocked in genetically modified mice lacking COX2 activity (Figure 1C) and when COX2 was inhibited (parecoxib, $10 \mathrm{mg} / \mathrm{kg}$ ) during the pain sessions (Figure 1D). To identify the cell type producing the critical prostaglandins, we next used the Cre/loxP system to delete Cox2 in specific cell types. Mice without COX2 in myeloid cells (Cox $2^{f l / f l} L y s M-C r e$; Figure 1E) or in brain endothelial cells (Cox $2^{f l / f l}$ Slco1c1-Cre mice; Figure $1 \mathrm{~F}$ ) displayed aversion comparable to that of littermates without Cre (WT mice). In contrast, mice without COX2 in neural cells (Cox$2^{f / f l} \mathrm{Nes}$-Cre mice), including peripheral and central neurons and glia, showed no avoidance behavior (Figure 1G).

Prostaglandin $\mathrm{E}_{2}$ is the principal proinflammatory prostanoid and an important regulator of nociceptive and systemic inflammatory responses $(7,9-11)$. We tested mice lacking mPGES-1, the inducible form of prostaglandin $\mathrm{E}$ synthase, in our pain model (mPges-1 KO mice). These mice displayed significantly lower aversion scores compared with their WT littermates (Figure 1H). Prostaglandin $\mathrm{E}_{2}$ binds to $4 \mathrm{G}$ protein-coupled receptors called $\mathrm{EP}_{1}$ to $\mathrm{EP}_{4}$ (encoded by Ptger1-4). We tested mice lacking $\mathrm{EP}_{1}$ or $\mathrm{EP}_{3}$ receptors, since these are strongly expressed in brain structures related to motivation $(12,13)$ and have been shown to be implicated in nociceptive processing (14-16). Mice lacking $\mathrm{EP}_{1} \mathrm{Rs}_{\text {(Ptger1 }}{ }^{-1}$ mice, referred to as EP1R KO mice) did not show any difference in 
A

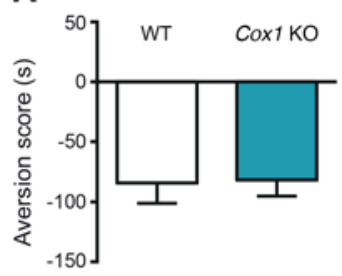

E

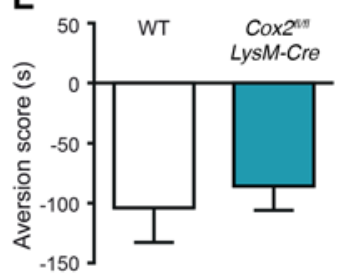

B

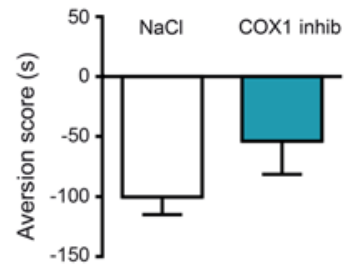

$\mathbf{F}$

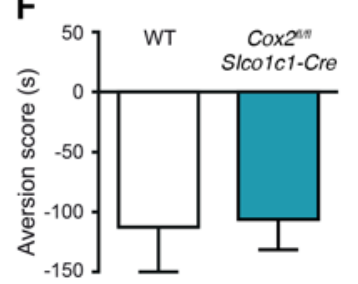

C

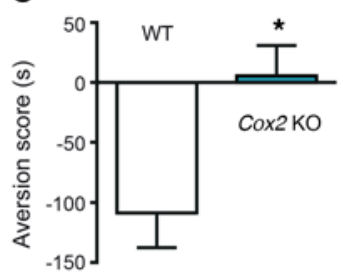

G

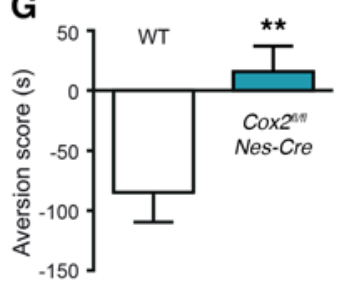

D
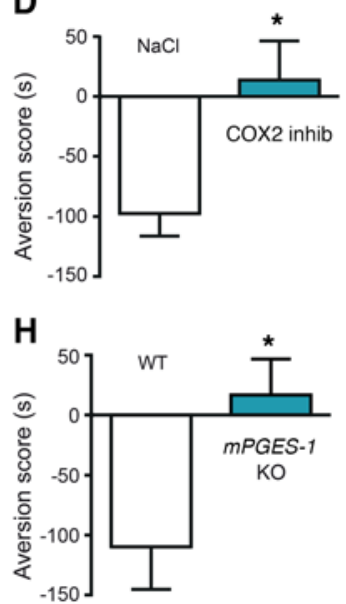

Figure 1. The affective component of inflammatory pain is dependent on prostaglandin E2 generated by COX2 in neural cells. (A-D) Conditioned place aversion induced by injection of diluted formalin in the dorsal part of the hind paws in mice lacking COX1 (Cox1 K0) (A), mice treated with a COX1 inhibitor (COX1 inhib) (sc560, 5 mg/kg, i.p.; B), mice lacking COX2 (Cox2 KO) (C), mice treated with a COX2 inhibitor (parecoxib, 10 mg/kg, i.p.; D), and corresponding control mice. (E) Aversion scores in mice lacking COX2 in myeloid cells (Cox $2^{f l / f l}$ LysM-Cre). See Supplemental Methods for calculation of aversion scores. (F and G) Aversion scores in mice lacking COX2 in brain endothelial cells (Cox $2^{f l / f l}$ Slco1c1-Cre) or mice lacking COX2 in neural cells (Cox2 fl/fl $\left.N e s-C r e\right)$. (H) Aversion is also blocked in mice lacking the inducible microsomal prostaglandin E synthase (mPCES-1 KO). ${ }^{*} P<0.05 ;{ }^{*} P<0.01$, Student's $t$ test.

aversion scores compared with WT littermates (Figure 2A). In contrast, Ptger3 knockout (EP3R KO) mice displayed no pain-induced aversion (Figure 2B).

$\mathrm{EP}_{3}$ receptors are expressed in many structures of the brain and the spinal cord. Given the important role of serotonin in the regulation of affective functions, the $\mathrm{EP}_{3}$ receptor expression in serotonergic neurons of the dorsal raphe nucleus and other serotonergic structures (12) is particularly interesting in the context of aversion. To explore the role of $\mathrm{EP}_{3}$ receptors on serotonergic neurons, we next used Ptger $3^{f l / f l}$ Sert-Cre mice (referred to as EP3R-SERTCre mice). In these mice, Ptger 3 is deleted by Cre expressed under control of the serotonin transporter (Sert) promoter. As expected, Cre expression was specific to serotonergic neurons, identified by labeling for tryptophan hydroxylase $(\mathrm{TpH})$ (Figure 2, C and D). Further, EP3R-SERTCre mice displayed a clear reduction of Ptger $3-\alpha$ mRNA in tissue punches from the dorsal raphe region, whereas no reduction was seen in the cortex or the hypothalamus/thalamus (Supplemental Figure 2A). Subsequently, we tested the EP3R-SERTCre mice in the conditioned pain-avoidance test. We observed a complete abrogation of the aversion in EP3R-SERTCre mice (Figure 2E). Further, aversion was completely blocked in mice lacking the serotonin transporter SERT (Figure 2F). The serotonergic system can be divided into 2 main parts (17). One is ascending, involved in affective processing, and is constituted by the dorsal and median raphe nuclei. The other is descending and modulates nociceptive processing in the spinal cord (17, 18). To determine whether the ascending serotonergic pathways mediated the aversion, we deleted Ptger 3 receptors in the region of the dorsal raphe nucleus. This was done by stereotaxic injection of Cre-expressing viral vectors (AAV5) into mice with floxed Ptger3. Mice with injections that affected the dorsal raphe nucleus (Figure 2G) without extending to the descending raphe nuclei showed blocked aversion (Figure $2 \mathrm{H}$ ). Collective- ly, these findings strongly suggest that ascending serotonergic transmission is critical for pain-induced aversion and that prostaglandin $\mathrm{E}_{2}$ can modulate such transmission by a direct effect on $\mathrm{EP}_{3}$ receptors on serotonergic neurons.

Since most $\mathrm{EP}_{3} \mathrm{R}$ splice variants are coupled to inhibitory $G$ proteins, we investigated whether $\mathrm{EP}_{3} \mathrm{R}$ activation elicits aversion by inhibition of serotonergic neurons. To test this, we used a designer receptor exclusively activated by designer drug-based (DREADD-based) chemogenetic approach (19) to maintain firing in serotonergic cells during the painful experience and monitored the aversive response of the mice. We injected viral vectors with Cre-dependent expression of Gq-coupled DREADDs (hM3Dq) or mCherry in SERT-Cre mice (AAVhM3Dq Sert-Cre and AAV-mCherry Sert-Cre mice, respectively; Figure 2I) and activated the cells by administration of clozapine $\mathrm{N}$-oxide (CNO) before the formalin injections. Strikingly, chemogenetic activation of serotonergic cells blocked the aversion (Figure 2J). Further, mice without $\mathrm{EP}_{3} \mathrm{Rs}$ on serotonergic cells, which normally display no pain-induced aversion, showed a normal aversion when their serotonergic cells were inhibited during the formalin-pain session (Figure 2, K and L). This was achieved by stereotaxic injections of AAVs encoding Gicoupled DREADDs (hM4Di) or mCherry in EP3R-SERTCre mice (AAV-hM4Di EP3R-SERTCre and AAV-mCherry EP3RSERTCre mice, respectively; Figure $2 \mathrm{~K}$ ).

Next, we investigated whether the prostaglandin-dependent pathwayidentified was specific to the affective componentofpain or whether it was necessary for all aspects of pain. We monitored acute formalin-induced nociceptive behaviors (lifting, shaking, and licking the injected paw) in mice subjected to pharmacological inhibition of COX2 in mPGES-1 KO mice and in EP3RSERTCre mice. Pharmacological inhibition of COX2, as well as lack of mPGES-1, markedly reduced the nociceptive behaviors in the second phase of the formalin test (Figure 3, A and 
A

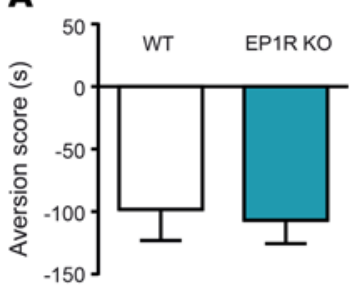

B

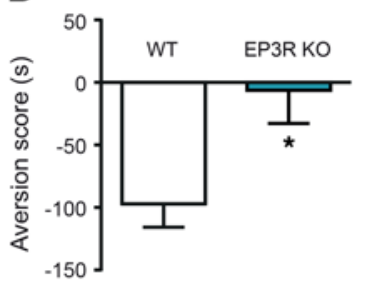

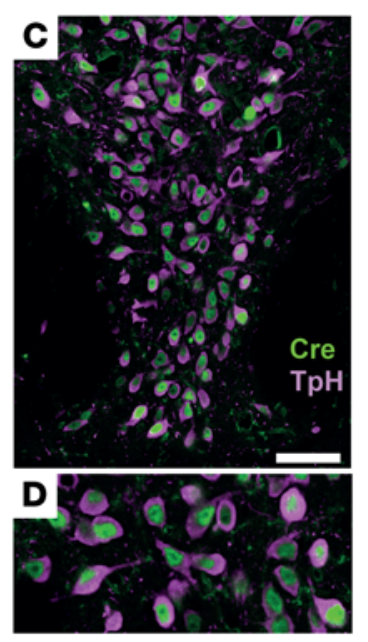

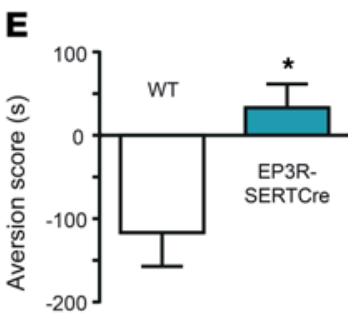

$\mathbf{F}$

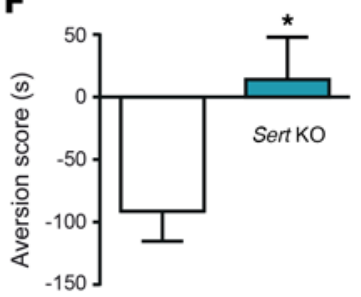

G

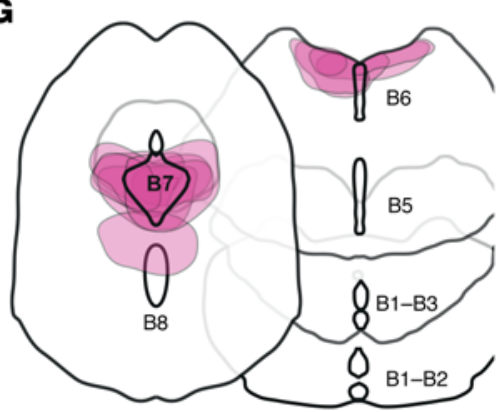

H

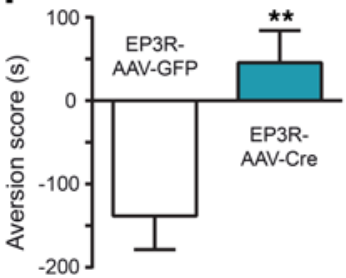

J

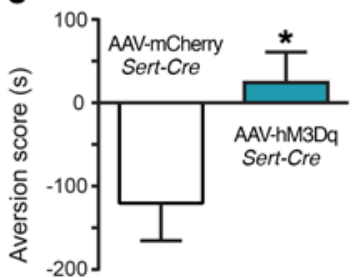

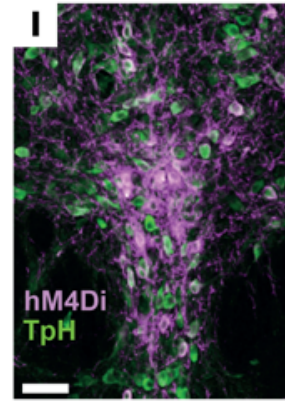

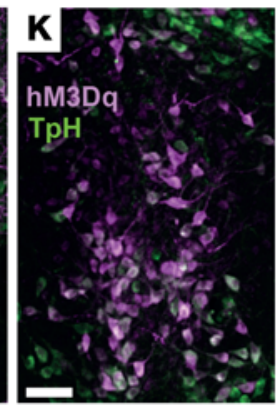

L

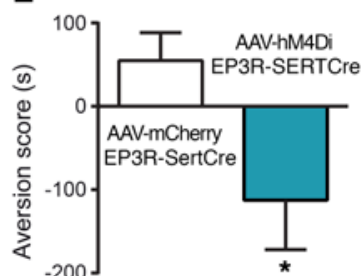

Figure 2. $E P_{3}$ receptors on serotonergic cells are critical for the affective component of pain. (A and $\left.\mathbf{B}\right)$ Aversion scores in mice lacking $E P_{1}$ receptors $(E P 1 R$ $\mathrm{KO})(\mathbf{A})$ or $\mathrm{EP}_{3}$ receptors (EP3R KO) (B). (C and D) Confocal micrographs from the dorsal raphe nucleus of a Sert-Cre mouse. Labeling for TpH, a marker for serotonergic neurons, is shown in purple, and Cre labeling is shown in green. $\mathbf{D}$ is a higher magnification of parts of $\mathbf{C}$. (E) Graph showing the aversive reaction to inflammatory pain in mice lacking $\mathrm{EP}_{3}$ receptors in serotonergic cells (EP3R-SERTCre) due to deletion driven by the Sert promoter. $(\mathbf{F})$ Aversion scores in control mice and mice lacking the serotonin transporter (Sert KO). (C) Plot of the areas transfected with the viral vector encoding Cre in Ptger $3^{f / f f}$ animals used in H. (H) Aversion scores in response to formalin-induced pain in mice with AAV-Cre-induced Ptger 3 deletion in the area of the dorsal raphe nucleus (B6-B7). (I) Expression of hM3Dq-mCherry in serotonergic cells of the dorsal raphe. (J) Aversion scores from mice in which serotonergic cells were chemogenetically activated (AAV-hM3Dq Sert-Cre + CNO) during the pain session and controls (AAV-mCherry/Sert-Cre + CNO). (K) Expression of $\mathrm{hM} 4 \mathrm{Di}$-mCherry in serotonergic cells of the dorsal raphe. (L) Aversion scores from EP3R-SERTCre mice in which serotonergic cells were chemogenetically inactivated (AAV-hM4Di EP3R-SERTCre + CNO) during the pain sessions and controls (AAV-mCherry EP3R-SERTCre + CNO). Scale bars: $50 \mu \mathrm{m}$ (C, I, and K); $30 \mu \mathrm{m}(\mathbf{D}) .{ }^{*} P<0.05 ;{ }^{*} P<0.01$, Student's $t$ test.

B), whereas they were unaffected in EP3R-SERTCre mice (Figure $3, \mathrm{C}$ and $\mathrm{D}$ ). This indicates that prostaglandin $\mathrm{E}_{2}$ modulates many components of pain, but that the effect on serotonergic transmission selectively controls the affective dimension. We also found a normal febrile response in EP3R-SERTCre mice (Figure 3E), indicating that responses driven by $\mathrm{EP}_{3} \mathrm{Rs}$ in nonserotonergic cells were intact $(20,21)$. Further, aversion induced by the $\kappa$ opioid receptor agonist U-50488 $(2.5 \mathrm{mg} / \mathrm{kg}$, i.p. $)$ was intact in EP3R-SERTCre mice (Figure 3F), and both Cox $2^{f / f l}$ Nes-Cre and EP3R-SERTCre mice displayed a robust aversion to thermal pain (Figure 3, G and H). EP3R-SERTCre mice also displayed normal sucrose preference, normal withdrawal latencies in the hot-plate test, and normal real-time avoidance of a hot floor (Supplemental Figure 2, B-D). Thus, prostaglandinmediated modulation of serotonergic signaling selectively controls aversion to inflammatory pain.

The finding that $\mathrm{EP}_{3}$ receptors on serotonergic neurons regulate the affective component of pain is in line with the central role of serotonin in affective functions (17) and may have relevance for the high comorbidity of pain and depression. Populations of serotonergic cells are modulated by aversive stimuli (22), including pain (23), as well as by rewarding stimuli (24). 
A

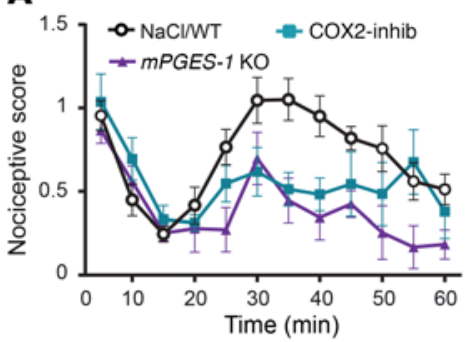

E

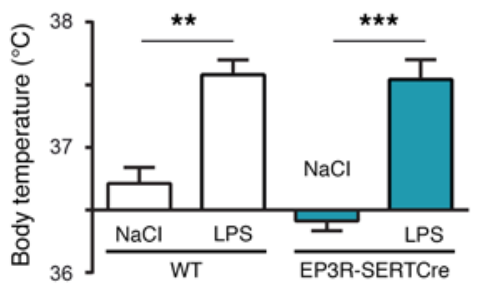

B

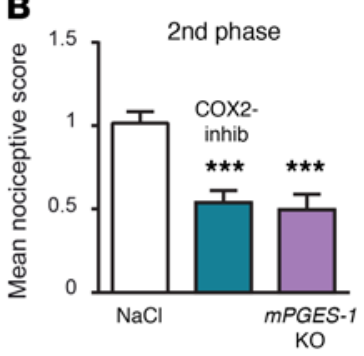

$\mathbf{F}$

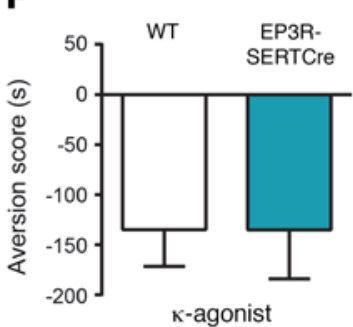

C

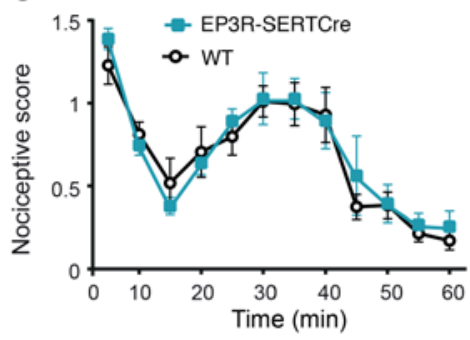

D

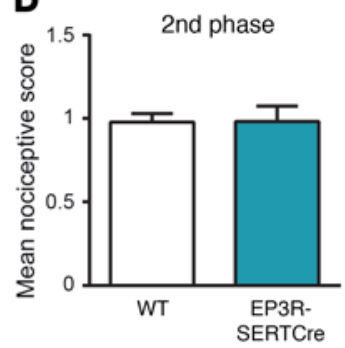

G

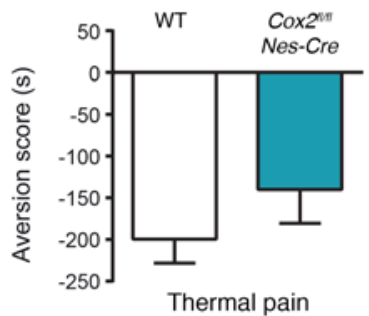

H

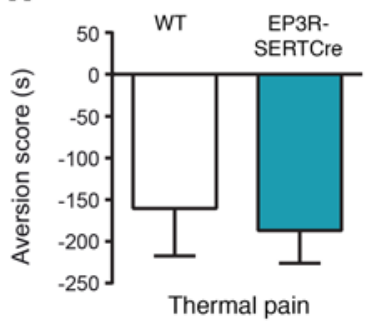

Figure 3. The involvement of $\mathbf{E P}_{3}$ receptors on serotonergic cells is specific for the affective component of pain. (A and $\left.\mathbf{B}\right)$ Nociceptive scores in mice given a COX2 inhibitor or mPCES-1 KO mice (parecoxib, $10 \mathrm{mg} / \mathrm{kg}$, i.p.; $n=9+7+6$ ). B shows means of the scores during the peak of the second phase of the formalin-induced pain (defined as 30-40 minutes after injection). See Supplemental Methods for calculation of nociceptive scores. (C and D) Corresponding data for mice lacking EP 3 receptors in serotonergic cells (EP3R-SERTCre; $n=5+5$ ). Note that the blunted response seen after COX2 inhibition cannot be seen in EP3R-SERTCre mice. (E) Febrile responses in WT and EP3R-SERTCre mice. Mean body temperatures 5 to 9 hours after an injection of lipopolysaccharide $(100 \mu \mathrm{g} / \mathrm{kg})$ are plotted. (F) Aversion scores in response to the $\kappa$ receptor agonist U50488 (2.5 mg/kg, i.p.). Note that EP3R-SERTCre mice display normal acute nociceptive responses and a normal U-50488-induced aversion. (G and $\mathbf{H}$ ) Conditioned place aversion induced by thermal pain in Cox $2^{f l / f l}$ Nes-Cre mice $(\mathbf{G})$ and EP3R-SERTCre mice $(\mathbf{H})$ and their WT littermates. ${ }^{* *} P<0.01 ;{ }^{* *} P<0.001$, Student's $t$ test $(\mathbf{B}, \mathbf{D}$, and $\mathbf{F}-\mathbf{H}) ;$ ANOVA (E).

Further, serotonergic neurotransmission mediates adaptive danger avoidance (25), can induce fear and anxiety (26), and regulates affective responses to stress $(27,28)$. The affective responses to stress are mediated by a transient increase in serotonin reuptake in the ventral striatum, leading to a local hyposerotonergic state (29). Prostaglandin-mediated inhibition of serotonergic cells could be another way of reducing serotonin levels in the ventral striatum. It is likely that the importance of ascending serotonergic neurons is not restricted to inflammatory pain. Thus, other types of pain may access the serotonergic cells by pathways not involving prostaglandins and $\mathrm{EP}_{3}$ receptors. We recently found that the aversion induced by systemic inflammation is mediated by prostaglandins (30). The characteristics of the aversive stimulus used in that study (LPS injected i.p.) are quite different from those of the stimulus used in this study. Thus, the systemic inflammation was presumably nonpainful and the signaling mechanisms involved the brain endothelium, COX1, and $\mathrm{EP}_{1}$ receptors on dopamine $\mathrm{D} 1$ receptor-expressing neurons in the striatum (30). In contrast, the aversion seen in this study was dependent on nociceptive input and could consequently be blocked by deletion of Trpa1 (Supplemental Figure 2E), the receptor activated by formalin on the primary afferents (31). Thus, prostaglandins are involved in the aversive signaling induced by both systemic inflammation and localized inflammatory pain, but different peripheral signaling routes, cyclooxygenases, EP receptor subtypes, and monoaminergic neuronal populations are involved.

In summary, we show that persistent inflammatory pain induces aversion through a mechanism involving neural prostaglandin synthesis and $\mathrm{EP}_{3}$ receptors on serotonergic cells. Where- as prostaglandins and serotonin modulate nociceptive processing at many levels, this mechanism selectively regulates the affective dimension of inflammatory pain.

\section{Methods}

Additional methods are provided in the Supplemental Methods.

Statistics. All the data are expressed as mean \pm SEM and were analyzed (in GraphPad Prism) using 2-tailed Student's $t$ tests, except for body temperature measurements, which were analyzed with 2-way ANOVA. $P<0.05$ was considered significant. The number of mice are specified in Supplemental Figure 1 or the figure legends.

Study approval. All experiments were approved by the Linköping Animal Care and Use Committee and followed national and international guidelines.

\section{Author contributions}

AKS, JZ, and DE were responsible for overall study design. AKS performed the behavioral/physiological experiments except for those involving $\mathrm{EP}_{1} \mathrm{R}-\mathrm{KOs}$ (AMK and $\mathrm{MF}$ ), fever (EM), or thermal pain and sucrose preference (NA, IF, and JZ). MF designed, implemented, and introduced others to the formalin conditioning protocol. Viral injections and histological validations were done by JZ. Quantitative PCR (qPCR) analysis was done by JZ, AKS, MJ, and IF. The manuscript was written by DE, AKS, and JZ. All authors were involved in the design of the parts of the study they executed, discussed the design and results, and commented on the manuscript.

\section{Acknowledgments}

This study was supported by the European Research Council, the Swedish Medical Research Council, the Knut and Alice 
Wallenberg Foundation, the Swedish Brain Foundation, and the County Council of Östergötland. The Sert-Cre line was from the Mutant Mouse Regional Resource Center and was originally donated by the GENSAT BAC transgenic project, funded by the National Institute of Neurological Disorders and Stroke (NINDS). We thank Harvey R. Herschman for providing the $\operatorname{Cox} 2^{f / f l}$ line, Markus Schwaninger for providing the Slco1c1-
CreER line, Anders Blomqvist for providing several mouse lines, Johan Jakobsson for input on viral vectors, and Johanna Karlsson for technical assistance.

Address correspondence to: David Engblom, Department of Clinical and Experimental Medicine, Linköping University, 58185 Linköping, Sweden. Phone: 46.101038448; E-mail: david.engblom@liu.se.
1. Craig AD. A new view of pain as a homeostatic emotion. Trends Neurosci. 2003;26(6):303-307.

2. Price DD. Psychological and neural mechanisms of the affective dimension of pain. Science. 2000;288(5472):1769-1772.

3. Navratilova E, Porreca F. Reward and motivation in pain and pain relief. Nat Neurosci. 2014;17(10):1304-1312.

4. Rainville P, Duncan GH, Price DD, Carrier B, Bushnell MC. Pain affect encoded in human anterior cingulate but not somatosensory cortex. Science. 1997;277(5328):968-971.

5. Johansen JP, Fields HL, Manning BH. The affective component of pain in rodents: direct evidence for a contribution of the anterior cingulate cortex. Proc Natl Acad Sci U S A. 2001;98(14):8077-8082.

6. Johansen JP, Fields HL. Glutamatergic activation of anterior cingulate cortex produces an aversive teaching signal. Nat Neurosci. 2004;7(4):398-403.

7. Chen L, Yang G, Grosser T. Prostanoids and inflammatory pain. Prostaglandins Other Lipid Mediat. 2013;104-105:58-66.

8. Ide S, et al. Opposing roles of corticotropin-releasing factor and neuropeptide $\mathrm{Y}$ within the dorsolateral bed nucleus of the stria terminalis in the negative affective component of pain in rats. J Neurosci. 2013;33(14):5881-5894.

9. Trebino CE, et al. Impaired inflammatory and pain responses in mice lacking an inducible prostaglandin E synthase. Proc Natl Acad Sci U S A. 2003;100(15):9044-9049.

10. Engblom D, et al. Microsomal prostaglandin E synthase-1 is the central switch during immune-induced pyresis. Nat Neurosci. 2003;6(11):1137-1138.

11. Ek M, Engblom D, Saha S, Blomqvist A, Jakobsson PJ, Ericsson-Dahlstrand A. Inflamma- tory response: pathway across the blood-brain barrier. Nature. 2001;410(6827):430-431.

12. Nakamura K, Li YQ, Kaneko T, Katoh H, Negishi M. Prostaglandin EP3 receptor protein in serotonin and catecholamine cell groups: a double immunofluorescence study in the rat brain. $\mathrm{Neu}$ roscience. 2001;103(3):763-775.

13. Kitaoka S, et al. Prostaglandin E2 acts on EP1 receptor and amplifies both dopamine D1 and D2 receptor signaling in the striatum. J Neurosci. 2007;27(47):12900-12907.

14. Minami T, et al. Characterization of EP receptor subtypes responsible for prostaglandin E2-induced pain responses by use of EP1 and EP3 receptor knockout mice. Br J Pharmacol. 2001;133(3):438-444.

15. Natura G, et al. Neuronal prostaglandin E2 receptor subtype EP3 mediates antinociception during inflammation. Proc Natl Acad Sci US A. 2013;110(33):13648-13653.

16. Bar KJ, et al. Changes in the effect of spinal prostaglandin E2 during inflammation: prostaglandin E (EP1-EP4) receptors in spinal nociceptive processing of input from the normal or inflamed knee joint. J Neurosci. 2004;24(3):642-651.

17. Dayan P, Huys QJ. Serotonin in affective control. Annu Rev Neurosci. 2009;32:95-126.

18. Cai YQ, Wang W, Hou YY, Pan ZZ. Optogenetic activation of brainstem serotonergic neurons induces persistent pain sensitization. Mol Pain. 2014;10:70.

19. Roth BL. DREADDs for neuroscientists. Neuron. 2016;89(4):683-694.

20. Lazarus M, et al. EP3 prostaglandin receptors in the median preoptic nucleus are critical for fever responses. Nat Neurosci. 2007;10(9):1131-1133.

21. Ushikubi F, et al. Impaired febrile response in mice lacking the prostaglandin $\mathrm{E}$ receptor sub- type EP3. Nature. 1998;395(6699):281-284.

22. Cohen JY, Amoroso MW, Uchida N. Serotonergic neurons signal reward and punishment on multiple timescales. Elife. 2015;4:e06346.

23. Schweimer JV, Ungless MA. Phasic responses in dorsal raphe serotonin neurons to noxious stimuli. Neuroscience. 2010;171(4):1209-1215.

24. Li Y, et al. Serotonin neurons in the dorsal raphe nucleus encode reward signals. Nat Commun. 2016;7:10503.

25. Amo R, et al. The habenulo-raphe serotonergic circuit encodes an aversive expectation value essential for adaptive active avoidance of danger. Neuron. 2014;84(5):1034-1048.

26. Marcinkiewcz CA, et al. Serotonin engages an anxiety and fear-promoting circuit in the extended amygdala. Nature. 2016;537(7618):97-101.

27. Land BB, et al. Activation of the kappa opioid receptor in the dorsal raphe nucleus mediates the aversive effects of stress and reinstates drug seeking. Proc Natl Acad Sci U S A. 2009;106(45):19168-19173.

28. Bruchas MR, et al. Selective p38 $\alpha$ MAPK deletion in serotonergic neurons produces stress resilience in models of depression and addiction. Neuron. 2011;71(3):498-511.

29. Schindler AG, et al. Stress produces aversion and potentiates cocaine reward by releasing endogenous dynorphins in the ventral striatum to locally stimulate serotonin reuptake. J Neurosci. 2012;32(49):17582-17596.

30. Fritz M, et al. Prostaglandin-dependent modulation of dopaminergic neurotransmission elicits inflammation-induced aversion in mice. J Clin Invest. 2016;126(2):695-705.

31. McNamara CR, et al. TRPA1 mediates formalin-induced pain. Proc Natl Acad Sci USA. 2007;104(33):13525-13530. 